OPEN ACCESS

Edited by:

Paolo Tonin,

Sant'Anna Institute, Italy

Reviewed by:

Maria Carmen Usai,

University of Genoa, Italy

Linda Daniela,

University of Latvia, Latvia

Alessandra Bardoni,

Eugenio Medea (IRCCS), Italy

*Correspondence:

Giuseppina Sgandurra

g.sgandurra@fsm.unipi.it

Specialty section: This article was submitted to

Neurorehabilitation,

a section of the journal

Frontiers in Neurology

Received: 31 August 2020 Accepted: 27 November 2020

Published: 20 January 2021

Citation:

Di Lieto MC, Pecini C, Brovedani P,

Sgandurra G, Dell'Omo M,

Chilosi AM, Guzzetta A, Perazza S,

Sicola E and Cioni G (2021) Adaptive

Working Memory Training Can

Improve Executive Functioning and

Visuo-Spatial Skills in Children With

Pre-term Spastic Diplegia.

Front. Neurol. 11:601148.

doi: 10.3389/fneur.2020.601148

\section{Adaptive Working Memory Training Can Improve Executive Functioning and Visuo-Spatial Skills in Children With Pre-term Spastic Diplegia}

\author{
Maria Chiara Di Lieto ${ }^{1}$, Chiara Pecini ${ }^{2}$, Paola Brovedani ${ }^{1}$, Giuseppina Sgandurra ${ }^{1,3 *}$, \\ Marta Dell'Omo ${ }^{1}$, Anna Maria Chilosi ${ }^{1}$, Andrea Guzzetta ${ }^{1,3}$, Silvia Perazza ${ }^{1}$, Elisa Sicola ${ }^{1}$ \\ and Giovanni Cioni ${ }^{1,3}$ \\ ${ }^{1}$ Department of Developmental Neuroscience, Istituto di Ricovero e Cura a Carattere Scientifico (IRCCS) Fondazione Stella \\ Maris, Pisa, Italy, ${ }^{2}$ Department of Education, Language, Interculture and Psychology, University of Florence, Florence, Italy, \\ ${ }^{3}$ Department of Clinical and Experimental Medicine, University of Pisa, Pisa, Italy
}

Pre-term spastic diplegia (pSD) due to periventricular leukomalacia is a form of cerebral palsy in which weaknesses in executive functions are reported beyond the core visuo-spatial deficits. The study aimed at improving executive functioning and visuo-spatial skills with an evidence-based training focused on working memory in children with pSD. The intervention study followed a stepped wedge design. 19 children with pSD (11 female and 8 male; age range: 4;1-13;1 years), mild to moderate upper limb impairment and Verbal Intelligence Quotient $(\mathrm{VIQ})>80$ participated to the study. The children were trained with a home-based adaptive working memory training (CogMed $\left.{ }^{\circledR}\right)$ over a 5-week period. The primary outcome measure was the CogMed Improvement index; pre- and post-test explorative neuropsychological assessment was conducted with a subset of tests from the NEPSY-II battery. Working memory training in children with pSD significantly improved trained working memory abilities (CogMed indices) as well as non-trained skills, such as visuo-spatial skills, inhibition of automatic responses and phonological processing. The results suggest that standard rehabilitation schedules for children with pSD should be integrated with trainings on executive functions.

Keywords: pre-term spastic diplegia, executive function, visuo-spatial function, neuropsychological training, cogmed working memory training

\section{INTRODUCTION}

Working memory (WM) is the ability to manipulate and update information maintained in memory for brief periods of time (1). It is important in several complex cognitive functions, such as academic learning, planning and organization of daily life activities. School-based activities, indeed, such as math and reading, depend on the ability to hold and integrate several instructions or information in mind (2-8). Working memory belongs to the family of top-down mental processes known as executive functions (EFs) $(9,10)$. Within a general pattern of shared but distinct EFs (11), the developmental model proposed by Diamond (7) is largely adopted to describe three main EF components: (i) inhibition, the ability to suppress automatic behaviors, memories and 
thoughts in favor of goal-appropriate responses, (ii) WM, the ability to actively manipulate relevant information in memory and (iii) cognitive flexibility, the ability to switch between two or more tasks, mental sets or response rules. Although it is wellaccepted that there are several processes within the EF domain (11), recent reviews of EF interventions highlight that especially WM and inhibition must be continually challenged as they are considered "tools for learning" [(12), page 363], favoring the development of other cognitive skills $(6,8,12,13)$.

Since EF deficits are typically found in children with Attention Deficit and Hyperactivity Disorder [ADHD, for a review see (14)] or traumatic brain injury (15), several studies suggest that EF impairment may be part of different neurodevelopmental disorders such as specific learning disabilities [for a meta-analysis see (16)], specific language impairment [for a review see (17)], intellectual disabilities $(18,19)$, autism spectrum disorders $(20)$ and cerebral palsy, including spastic diplegia $(21,22)$.

Spastic diplegia is a form of cerebral palsy (CP) in which both sides of the body are involved, with a predominance to the lower limbs (23). It commonly occurs in preterm born children (pre-term spastic diplegia, pSD) and it is generally due to periventricular leukomalacia, a form of white matter brain injury typically affecting neural pathways lying close to the lateral ventricles, as the corticospinal tract and the optic radiations (24-28). Children with pSD consistently present with impaired non-verbal intelligence and visuo-perceptual and visuo-spatial abilities, while general verbal skills, as reflected by verbal Intelligence Quotient and Indices, are generally spared $(26,28-30)$. Beyond visuo-spatial deficits and impaired nonverbal intelligence, weak EFs have been also reported in children with $\mathrm{pSD}$, such as the inability to quickly process, maintain, update and inhibit information $(21,31-36)$. This might be due to the involvement of white matter associative fibers altering brain structural connectivity $(24,26-28)$, as shown, for example, in children with unilateral periventricular leukomalacia where the altered connectivity to the anterior cingulate cortex is strongly related to EF deficits (37). In a previous study from our group (36), a multilevel organization of the neuropsychological profile in children with $\mathrm{pSD}$ was suggested. Beyond the common core visuo-spatial and sensory-motor deficits, when pSD was associated with thinning of the anterior/middle corpus callosum, impairments in attention and EFs seemed to act as additional factors in further affecting visuo-spatial, sensori-motor and social skills.

Despite the complex neuropsychological deficit patterns of $\mathrm{pSD}$, as for other forms of CP, the majority of interventions for $\mathrm{CP}$ have been mainly focused on body motor functions and less is known on their effects on cognition, academic achievements and daily life skills [for a review of intervention research see (38)]. Some studies reported, in children with CP, intervention-related improvements in cognitive skills $(39,40)$ or in psychological wellbeing and social participation $(39,41-43)$. Evidence of training effects specifically targeting EFs is only relatively recent and scant, and has been reported for unilateral spastic CP $(44,45)$ but no studies to date have been performed in children with pSD.

One of the most widely used EF training programs in the scientific literature is the CogMed Working Memory Training
(RoboMemo $^{\circledR}$, CogMed Cognitive Medical Systems AB, Stockholm, Sweden), an evidence-based tele-rehabilitation software, comprising several intensive visuo-spatial and verbal working memory exercises that automatically adapt the level of difficulty to the individual child's performance. In healthy adults, training effects of CogMed have been linked to significant increases of activity in parietal and pre-frontal regions on WM task-related functional Magnetic Resonance Imaging (fMRI) (46-48), supporting training-induced plasticity of the neural systems underlying WM. CogMed's home-based videogame is currently applied to different clinical populations. It has been shown to improve WM skills in children with ADHD (49-52), acquired brain injury (53) as well as in pre-term-born children. In the latter, a population at higher risk for neurodevelopmental delay and CP (54), five non-randomized trials were performed (55-59), all using the CogMed platform. Benefits of the intervention were found in trained WM tasks as well as in untrained memory tasks, in very-low and extremely-low birth weight children, especially in visuo-spatial memory (spatial span and memory for faces tasks) and in verbal short and long term memory tasks (55-57). Beyond the generalized memory improvement, the effect on untrained cognitive processes, such as auditory attention and phonological awareness found by Grunewaldt et al. (56), was not confirmed by another study, most likely due to the methodological differences between the studies (58).

Generalization effects of CogMed training are still debated as some studies have found positive effects $(13,49,53,60-64)$ while others have reported no generalized enhancements of untrained skills $(59,65)$. The few follow-up studies available report a partially sustained training effect after 3 and 12 months $(65,66)$.

The present study was aimed at determining the effects of WM training in children with pSD due to periventricular leukomalacia. As working memory represents a fundamental component of EF, it was hypothesized that improving WM may have cascade effects on the deficits classically reported in this clinical population, such as visuo-spatial or sensori-motor impairment. CogMed was chosen as it allows auto-adaptive and intensive exercises at home, reducing the number of hospital visits, which are very frequent for children with pSD. This study replicated the methods and procedures of a previous study on CogMed training on pre-term born children without neuroanatomical lesions (56). The study has been registered with ClinicalTrials.gov, number NCT02342990, on January 20, 2015.

\section{MATERIALS AND METHODS}

\section{Participants}

Sample size was calculated by expected effect size method (67) by $\mathrm{G}^{*}$ Power 3 program (68). A total sample size of 19 children was selected, based on the data from 53 , showing an effect size on the primary outcome measure $d=0.8$, alpha $=0.05$ and power $=0.95$ for a dependent-sample t-test (critical $t=1.73$ ) (69).

Nineteen children ( 11 females, 8 males) with pSD (mean age $7 ; 3$ years, SD: 2;4 range 4;1-13;1 years) and a mean gestational age at birth of 31 weeks (range: 28-35 weeks) were selected from a group of 30 children with a $\mathrm{CP}$, recorded as spastic 
diplegia according to Bax et al. (70), recruited from March 2014 to November 2015 at the Department of Developmental Neuroscience of IRCCS Fondazione Stella Maris. Children were selected according to the following inclusion criteria: (a) neuroradiological diagnosis of periventricular leukomalacia documented at brain MRI performed after age 2 years (by images or on neuroradiological reports); (b) mild to moderate functional upper limb impairment (from level I to III) at the Manual Ability Classification System- MACS (71); (c) absence of drug-resistant epilepsy; (d) absence of a psychiatric disorder diagnosis or sensory deficits that preclude testing; (e) Verbal IQ $>80$, as assessed in the last year prior to recruitment by WPPSI-III (72), WISC-III (73) or WISC-IV (74). The majority of children (17 out of 19) had significantly higher verbal intelligence than non-verbal. After the enrollment, the children were randomly split into two groups (Cluster A, $n=10$ and Cluster $\mathrm{B}, n=9$ ), for sequential rollout of the training. All children were native Italian speakers of European ethnicity and followed care as usual motor rehabilitation. A subset of the children included were part of a previous study (36).

The research project was approved by the Ethical Committee of the Institute ( $\left.n^{\circ} 13 / 2013\right)$. Written consent for participation was obtained from all participants' parents who also gave informed consent to publication of results.

\section{Motor and Visual Assessment}

The Gross Motor Classification System (GMCS) (75) was used to determine gross motor skills. Children were classified in five motor levels: walk without restriction (level I); walk without assistive devices but limitation in walking outdoors (level II); walk with assistive mobility devices (level III); self-mobility with limitations (level IV); self-mobility is severely limited even with use of assistive technology (level V). Manual ability was classified according to the MACS (71). Children were classified according to five motor levels: handles objects easily and successfully (level I); handles most objects but with somewhat reduced quality and/or speed of achievement (level II); handles objects with difficulty; needs help to prepare and/or modify activities (level III); handles a limited selection of easily managed objects in adapted situations (level IV); does not handle objects and has severely limited ability to perform even simple actions (level V). Visual functions were derived on the basis of chart report data from the Vision Laboratory of our Department and were assessed for the presence of the following visual deficits: stereopsis impairment, deficits in ocular motility, visual field or visual acuity. Children were classified as follows: normal, absence of deficits; mildly impaired, one or two visual deficits; severely impaired, three or more deficits.

Motor, visual, and cognitive functions of the sample are reported in Table $\mathbf{1}$.

All children with pSD had more impaired lower limbs (GMFCS, classification ranging from Level I to IV) than upper (MACS, inclusion criterion). Visual functions were mildly impaired in the majority of children (14/17).

\section{Intervention Program}

CogMed Working Memory Training (RoboMemo ${ }^{\circledR}$, CogMed Cognitive Medical Systems AB, Stockholm, Sweden) contains a variety of computerized, game-format tasks that are homebased and auto-adaptive; that is, for each task, the level of difficulty is adjusted automatically to the WM span of the child. This training is available in three on-line versions depending on the child's age. Sixteen children used the school age version (CogMed RM), while three (S3, S8, and S17 in Table 1) used the pre-school version (CogMed JM). Pre-school children who read letters and numbers, at a preliminary qualitative assessment, used the school-age CogMed version. CogMed RM includes 12 visuo-spatial and verbal tasks, eight tasks are provided for each training session for $45 \mathrm{~min}$ a day; CogMed JM consists of seven visuo-spatial and verbal tasks for $20 \mathrm{~min}$ a day. A training period of 5 weeks, for a total of 25 sessions, was performed by each child at home. A certified coach (MCDL) introduced the CogMed program to the child and his/her family, establishing with them reward systems, goals and treatment planning and followed the training progress weekly calling the families to give advice based on the uploaded results. After the training, two indices were automatically provided by the program: CogMed improvement index, to measure working memory improvement, and CogMed progress indicators, which assesses visuo-spatial and verbal WM span [adapted from the Adaptive Working Memory Assessment; (76)]. For a detailed description of CogMed Working Memory Training see www.cogmed.com/program.

\section{Study Design}

As shown in Figure 1, the Stepped Wedge randomized trial design (77) adopted, was the same design previously used by Grunewaldt et al. (56) to study the CogMed effects in a group of premature pre-school children. Thus, the children were randomly split into two groups (Cluster A, $n$ $=10$ and Cluster $\mathrm{B}, n=9$ ), for sequential rollout of the training. Both Clusters were assessed with neuropsychological tests from the NEPSY-II at time point T0. Then children in Cluster B immediately started CogMed training, while those in Cluster A did not receive any training in the same period. Six/seven weeks later, all children (Cluster A and B) were retested (time point T1). At T1 Cluster A started CogMed training and 6/7 weeks later was retested at time point $\mathrm{T} 2$.

Test-retest effect was calculated comparing performance at T0 and $\mathrm{T} 1$ time points in Cluster A. The training effect was evaluated comparing pre- and post- training performance at NEPSY-II in all children (both Cluster A and Cluster B), controlling training differences between Clusters.

\section{Primary Outcome Measure}

The primary outcome measure was the CogMed Improvement index provided by the program, as it is largely used in literature and is representative of the main target of the training, that is, changes in working memory span $(53,56,78)$. It is calculated by subtracting the Start Index (the mean of the three best accurate trials on days 2 and 3) from the Max Index (the mean of the three best accurate trials during the training period). The mean CogMed Improvement Index for children aged 7-17 years is 27 (normal range 14-40). For further details about the 
TABLE 1 | Clinical characteristics of the study group of children with pSD.

\begin{tabular}{|c|c|c|c|c|c|c|c|c|}
\hline & Sex & GA & Age $(y ; m)$ & \multicolumn{2}{|c|}{ Motor function } & Visual function deficit & \multicolumn{2}{|c|}{ Intelligence } \\
\hline S1 & M & 28 & $9 ; 0$ & III & $\|$ & Mild & 102 & 85 \\
\hline S2 & M & 28 & $6 ; 0$ & $\|$ & $\|$ & NA & 88 & 93 \\
\hline S3 & $\mathrm{F}$ & 29 & $4 ; 1$ & III & 1 & NA & 106 & 76 \\
\hline S5 & $\mathrm{F}$ & 29 & $5 ; 1$ & IV & III & Mild & 114 & 104 \\
\hline S6 & $\mathrm{F}$ & 29 & $7 ; 0$ & $\|$ & 1 & Mild & 94 & 95 \\
\hline S7 & M & 35 & $6 ; 1$ & III & 1 & No & 92 & 61 \\
\hline S8 & $\mathrm{F}$ & 31 & $5 ; 1$ & $\|$ & $\|$ & No & 100 & 82 \\
\hline S9 & $\mathrm{F}$ & 32 & $8 ; 0$ & $\|$ & III & Mild & 82 & 58 \\
\hline S11 & M & 31 & $8 ; 1$ & $\|$ & $\|$ & Mild & 104 & 87 \\
\hline S12 & $\mathrm{F}$ & 32 & $9 ; 1$ & III & $\|$ & Mild & 102 & 100 \\
\hline $\mathrm{S} 13$ & $\mathrm{~F}$ & 32 & $6 ; 1$ & IV & III & Mild & 100 & 89 \\
\hline S14 & M & 30 & $11 ; 0$ & $\|$ & $\|$ & Mild & 103 & 59 \\
\hline S15 & $\mathrm{F}$ & 32 & $6 ; 0$ & $\|$ & $\|$ & No & 100 & 80 \\
\hline S16 & $\mathrm{F}$ & 34 & $13 ; 1$ & $\|$ & $\|$ & Mild & 98 & 93 \\
\hline S17 & $\mathrm{F}$ & 32 & $4 ; 1$ & $\|$ & II & Mild & 112 & 91 \\
\hline S18 & $M$ & 32 & $7 ; 8$ & $\|$ & $\|$ & Mild & 100 & 62 \\
\hline S19 & $\mathrm{F}$ & 28 & $9 ; 7$ & III & III & Mild & 99 & 89 \\
\hline Mean & & 31.4 & $8 ; 0$ & & & & 102.0 & 83.3 \\
\hline$S D$ & & 1.7 & $3 ; 0$ & & & & 4.2 & 14.0 \\
\hline
\end{tabular}

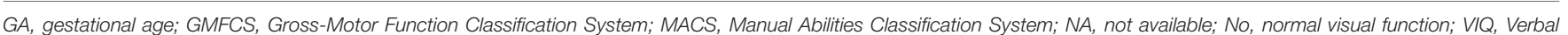

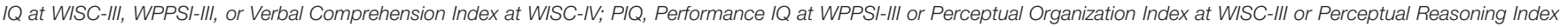
at WISC-IV.

training intervention and algorithm see CogMed JM and RM; www.cogmed.com and Klingberg et al. (79).

\section{Explorative Outcome Measures}

The CogMed progress indicators assess visuo-spatial and verbal WM span with two tests, Working Memory and Following Instructions, presented by the program at the beginning, the middle and the end of the training. The Working Memory test requires the child to identify a different shape from a set of three and remember its location; in the Following Instruction test the child listens to a set of instructions and then clicks on or drags objects seen on the screen in a specific order. Both tests scores are expressed in span scores.

The CogMed rating scale questionnaire was filled out by the parents as it is aimed at monitoring the child's behavior before and after the training period. The questionnaire collects quantitative data, expressed as raw scores, on inattention, hyperactivity and impulsivity. This questionnaire was filled only by parents of school-aged children, as provided by CogMed.
For pre- and post- neuropsychological evaluation, a subset of tests selected from the NEPSY-II battery was chosen (80), with particular attention to those subtests found impaired in a previous study in children with pSD (36). They tapped the following neuropsychological areas: executive function and attention, manual planning, visuo-spatial memory and visuospatial processing. Performance was expressed as raw scores, which, if not otherwise specified, were referred to response accuracy, that is the number of correct responses given for each subtest.The following subtests comprised the Executive function and attention assessment:

- Visual Attention: visual search task requiring to cross out one or two targets among a variable number of distractor stimuli;

- Auditory Attention and Response Set: sustained auditory attention task requiring to shift and update new and complex set of rules involving the inhibition of previously learned responses;

- Inhibition: task requiring to inhibit automatic responses in favor of novel responses and to switch between response types. The test is divided into three conditions: naming, inhibition, and switching, but only the inhibition condition was reported. 


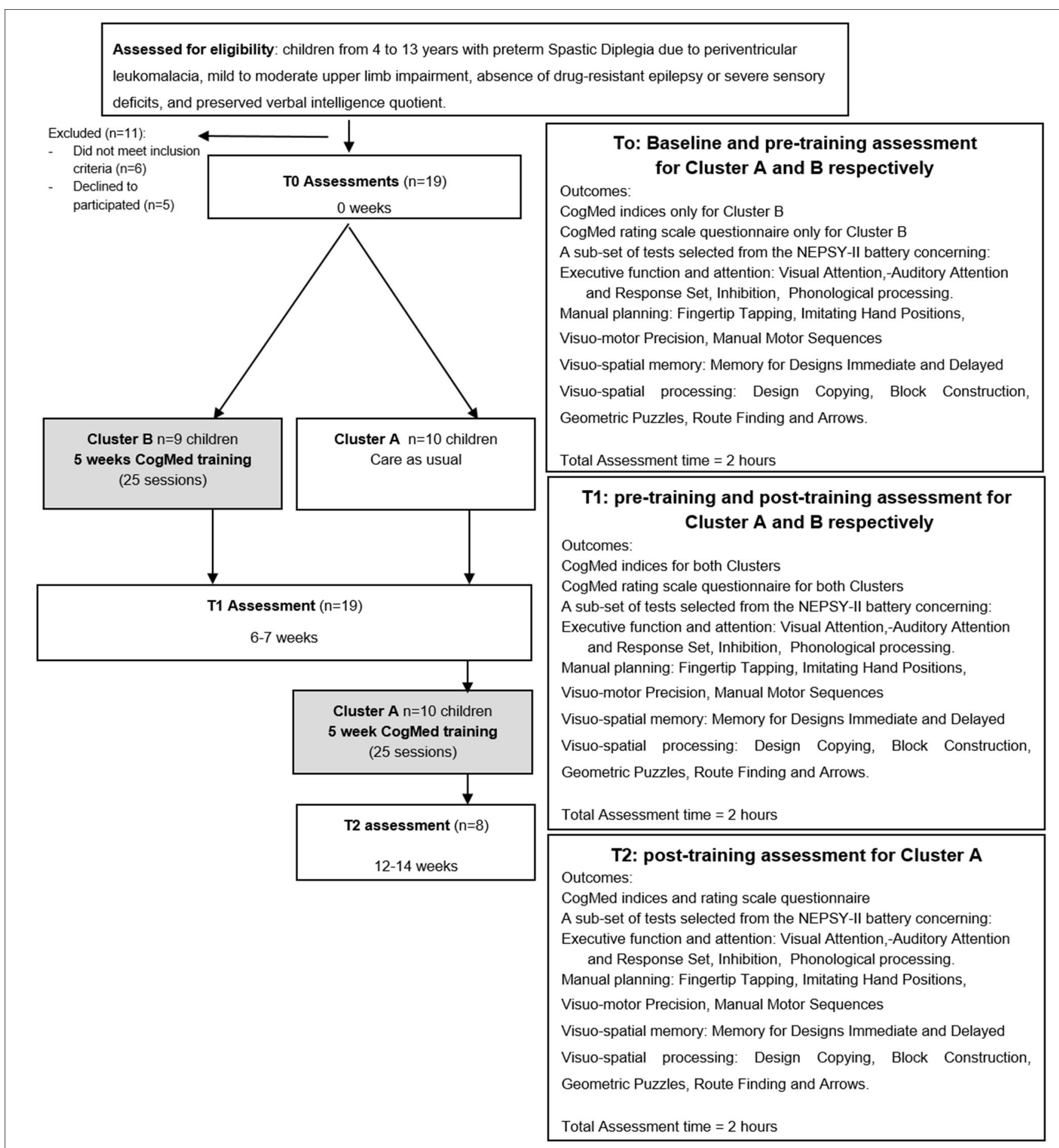

FIGURE 1 | CONSORT flow chart of the study.

Both accuracy (number of errors) and speed are recorded for each condition;

- Phonological processing: phonemic awareness task requiring to identify pictures corresponding to given word segments and to create new words by omitting or substituting a syllable or a phoneme.
For the Manual planning assessment, the following subtests were administered:

- Fingertip Tapping: tasks requiring to imitate a series of finger movements (single and sequences) with the dominant and non-dominant hand. Speed is recorded; 
- Imitating Hand Positions: visuo-motor planning task requiring to imitate finger positions;

- Visuo-motor Precision: visuo-motor integration task requiring to draw a line following paths of different widths and spatial complexity. Both accuracy (number of errors) and speed are measured;

- Manual Motor Sequences: visuo-motor planning task requiring imitation of a series of hand movements.

The Visuo-spatial memory assessment comprised the following subtests:

- Memory for Designs: visuo-spatial memory task requiring to identify form and position of an abstract design on a grid with 4-10 distractors. Content (visual form recognition) and spatial (localization) scores are obtained. Memory for Design Delayed is administered 15-25 min later.

For assessing Visuo-spatial processing, the following subtests were administered:

- Design Copying: visuo-motor integration task requiring to copy geometric figures of increasing complexity;

- Block Construction: constructional praxis task requiring imitation of three-dimensional block constructions of increasing complexity starting from either a three- or a two-dimensional model;

- Geometric Puzzles: mental rotation task requiring to recognize rotated geometric shapes among a series of distractors.

- Route Finding: visual spatial relations and directionality task requiring to find the house, previously shown in a schematic map, in a larger map with other houses and streets.

- Arrows: judgement of line orientation task requiring to find the arrow(s) pointing to the center of a the target in an array of arrows arranged around the target.

\section{Statistical Analysis}

The Statistical Package for Social Sciences, version 17.0 (IBM SPSS Statistics, IBM Corporation, Armonk, NY) was used for statistical analyses.

Normality of distributions was verified by Shapiro-Wilk's test and $t$-tests or non-parametric tests were used according to normal/non-normal distributed data or to continuous/ordinal variables, respectively.

To verify the absence of clinical and performance differences at pre-training assessment and to examine test re-test effect within Cluster A, two-tailed unpaired $t$-tests were conducted on pre-training performance between Cluster A and Cluster B and two-tailed paired $t$-tests within Cluster A.

To test the training effect on primary and explorative outcome measures, Mixed ANOVAs, with Cluster as between-subject factor (A vs. B) and time as repeated factor were performed on the primary outcome measures (CogMed indices) and on the neuropsychological subtest at NEPSY-II. For multiple comparisons, the Bonferroni's correction was applied. Moreover, to determine the effect size, Cohen's $d$ were calculated by $\mathrm{G}^{*}$ Power 3 program (68).

To verify change within CogMed progress indicators and questionaires, Wilcoxon Signed Rank test were performed comparing performance across the beginning, the middle and the end of the training in the CogMed progress indicators and between pre- and post- training in the CogMed rating scale questionnaire. To describe the relationship between clinical factors and training effects, Parametric or non-parametric bivariate correlations between clinical characteristics (VIQ, PIQ, chronological age, gestational age, GMFCS, and MACS) and CogMed Improvement index or the degree of improvement for each neuropsychological subtest were performed.

\section{RESULTS}

\section{Neuropsychological Characteristics of the Two Clusters at Pre-training Assessment}

There was no difference in chronological age $(t(17)=-1.2$, ns), gestational age $(t(17)=-1.4, \mathrm{~ns})$ and gender $\left(\chi^{2}(1)=2.7, \mathrm{~ns}\right)$ between the two Clusters.

At T0, no significant differences between Cluster $A$ and Cluster B were found in verbal and non-verbal intelligence $(t(17)$ $=-0.6$, ns; $t(17)=-0.2$, ns respectively) and in GMFCS $\left(\chi^{2}(3)\right.$ $=1.6, \mathrm{~ns})$. No differences between the two Clusters were found in Start CogMed index $(t(15)=-0.3$; ns) nor in any other NEPSY-II neuropsychological subtest at pre-training assessment, except for Auditory Attention $(t(15)=-2.4, p<0.05)$ and Design Copy $(t(16)=-2.8, p<0.05)$ subtests, which, thus, were excluded from further analysis.

\section{Training Effects on the CogMed Indices}

Two children (S7 and S10), included in Cluster A, did not complete the training due to inconsistent family compliance and thus their performance was used only to verify practice test-retest effects. All the other children $(n=17)$ completed the 25 -day training period and were tested at all scheduled time points.

As shown in Table 2, the Max Index was significantly higher than the Start Index $\left(F_{(1,15)}=52.72, p<0.001\right)$, without significant Cluster's effect $\left(F_{(1,15)}=0.26, p>0.05\right)$ and Cluster $\times$ Time effect $\left(F_{(1,15)}=0.55, p<0.05\right)$, and a large effect size was found $(d=1.29)$. The mean Improvement Index, that did not correlate with the Start and Max indices $(r(17)=0.4$, ns), was indeed higher (mean 25.2; SD 13.9; range between 8 and 52) than the improvement cut-off value (cut off $>14$ ).

The span scores in the Following Instruction test were significantly higher at the last session with respect to both the beginning $(Z=-2.8 ; p<0.005)$ and the middle $(Z=-2.3, p<$ $0.05)$ sessions. The span scores in the Working Memory tests and the behavioral profile at the parent rating scales (filled out for the sub-sample of children who performed the school age CogMed version, $n=14$ ) did not significantly change after the training (Wilcoxon signed ranks tests $Z$ from -0.14 to -1.9 , ns).

\section{Training Effects on Neuropsychological Measures}

Within Cluster A, a test-retest effect was found in Inhibition accuracy $(t(8)=3.2, p<0.05)$, Finger Tapping $(t(7)=3.1, p<$ $0.05)$, Manual Motor Sequences $(t(9)=-2.6, p<0.05)$. Thus, in order to avoid test-retest biases, the scores of these subtests were not used to test the training effects. 
TABLE 2 | CogMed working Memory indices.

\begin{tabular}{lccc}
\hline & Start Index & Max Index & Improvement Index \\
\hline S1 & 64 & 83 & $19^{\star}$ \\
S2 & 73 & 125 & $52^{\star}$ \\
S3 & 33 & 41 & 8 \\
S4 & 48 & 63 & $16^{\star}$ \\
S5 & 54 & 76 & $22^{\star}$ \\
S6 & 42 & 78 & $35^{\star}$ \\
S8 & 39 & 54 & $14^{\star}$ \\
S9 & 67 & 80 & $14^{\star}$ \\
S11 & 61 & 77 & $16^{\star}$ \\
S12 & 77 & 101 & $24^{\star}$ \\
S13 & 66 & 79 & 12 \\
S14 & 69 & 95 & $26^{\star}$ \\
S15 & 55 & 89 & $34^{\star}$ \\
S16 & 78 & 128 & $49^{\star}$ \\
S17 & 45 & 67 & $22^{\star}$ \\
S18 & 36 & 52 & $16^{\star}$ \\
S19 & 51 & 101 & $50^{\star}$ \\
Mean & $\mathbf{5 6 . 3}$ & $\mathbf{8 1 . 7}$ & $\mathbf{2 5 . 2}$ \\
(SD) & $(\mathbf{1 4 . 4 )}$ & $\mathbf{( 2 3 . 6 )}$ & $(\mathbf{1 3 . 9 )}$ \\
\hline
\end{tabular}

*Significant improvement ( $\geq 1$ SD from mean).

Mean and SDs for each NEPSY-II subtest, together with the mixed ANOVAs results are presented in Table 3. Within the executive function and attention subtests, significant improvements were found in Inhibition speed, with a moderate effect size, and in Phonological Processing with a small effect size. At the Memory for Design subtest (immediate condition) better performance at the end of the training was found with a moderate effect size, which was non significant after Bonferroni's Correction. Among the visuospatial processing subtests, significant improvements were found in Block Construction with a large effect size. No signficant Cluster effect were found, and Cluster $\times$ Time effect were found only in Phonological Processing subtests, which already showed almost significant differences between Clusters $(p=0.06)$ at pre-training assessment. A small minority of the children were unable to complete the neuropsychological assessment for clinical reason. The total number of children for each subtest is indicated in Table 3 under the heading "number of children who improved".

No significant correlations between CogMed Improvement index and verbal $(r(17)=-0.3$, ns) or non-verbal $(r(17)=0.4$, ns) intelligence levels were found. Chronological age positively correlated with Start and Max indices $(r(17)=0.6, p<0.01 ; r(17)$ $=0.6, p<0.01$, respectively), but not with Improvement index $(r(17)=0.40, \mathrm{~ns})$ and no correlations were found between other clinical characteristics and CogMed indices.

Concerning the relationship between clinical characteristics and improvements in the neuropsychological profile, GMFCS level was positively correlated with the immediate Memory for design $(\operatorname{rho}(17)=0.63, p<0.005)$ and negatively with the
Arrows $(\operatorname{rho}(14)=-0.70, p<0.01)$ subtests. Performance IQ was positively correlated with the improvement in Inhibition Speed $(r(15)=0.6, p<0.005)$ subtest, while verbal IQ was negatively correlated with Response Set $(r(8)=-0.9, p<0.01)$ and Imitation hand position $(r(15)=0.6, p<0.05)$ subtests. No correlations were found between gestational age and MACS levels and any neuropsychological subtests.

\section{DISCUSSION}

The main finding of our study is the demonstration that, in children with pSD, a home-based and self-adaptive WM training can improve targeted WM abilities as well as other non-targeted neuropsychological functions, such as visuo-spatial processing, inhibition, and phonological processing. In agreement with previous studies conducted in different clinical populations, we showed, for the first time, a direct effect of the training on WM abilities in children with pSD. This effect translates into large and significant improvements in CogMed indices and in an more active WM task requiring to maintain and process information in memory during a fine motor task, abilities called for the Following Instruction test. Thus, children with pSD increased both memory span, that is the number of units maintained in memory, and updating, the ability to control and actively manipulate information held for a short time in memory. These results, in agreement with Diamond's recommendation of continually challenging WM (6), suggest that an intensive and automatically adjusted training may be proposed to children with pSD to improve both storage and rapid updating. No significant differences emerged between pre- and post- training assessment at the CogMed rating scale questionnaires. We can speculate that given these questionnaires were tailored for children with $\mathrm{ADHD}$, they may not be sufficiently sensible to detect changing in executive function and attention difficulties associated to a neurological condition. Our findings showed a generalization of the CogMed effects to other neuropsychological processes not directly targeted by the training, some of which represent areas of weakness in children with $\mathrm{pSD}$, in particular visuo-spatial and executive functions. Significant improvements, indeed, were found in visuo-spatial tasks requiring visuo-construction abilities and in executive functioning, in terms of increased speed in inhibition and in improvements in phonological WM. These results are in agreement with several studies documenting CogMed cascade effects on untrained skills in everyday functions and on the core deficit of a certain neurodevelopment disorder [for a systematic review $(13,81)$ ].

Although these findings support, as highlighted by some authors (82), that the generalization effects of a WM training tend to mainly involve the components within the EF domain directly engaged in the training, they provide new and relevant insights for implementing cognitive rehabilitation strategies in children with pSD. In fact, WM is a transversal cognitive function, important for reasoning, comprehension and learning, which may influence cognition across-the-board and induce cascade improvements on several neuropsychological processes $(83,84)$. The finding of transfer effects to other impaired 
TABLE 3 | Comparison between pre- and post- training performance at single subtests.

\begin{tabular}{|c|c|c|c|c|c|c|c|c|c|c|}
\hline Outcome & & $\begin{array}{l}\text { Pre-training } \\
\text { Mean (SD) }\end{array}$ & $\begin{array}{l}\text { Post-training } \\
\text { Mean (SD) }\end{array}$ & $\boldsymbol{F}$ & df & $p$ & $\begin{array}{l}\text { Cluster's } \\
\text { effect } \\
\text { (p) }\end{array}$ & $\begin{array}{l}\text { Cluster } x \\
\text { Time effect } \\
\text { (p) }\end{array}$ & Cohen's d & $\begin{array}{c}\text { n. of } \\
\text { children } \\
\text { improved }\end{array}$ \\
\hline & Response Set & $27.7(8.5)$ & $30.4(6.4)$ & 5.61 & 1,6 & 0.065 & 0.757 & 0.065 & 0.3 & $4 / 7$ \\
\hline & Inhibition Speed & $137.6(48.7)$ & $122.9(44.0)$ & 18.08 & 1,13 & $0.001^{*}$ & 0.596 & 0.852 & 0.3 & $13 / 15$ \\
\hline \multirow[t]{2}{*}{ Visuo-spatial memory } & Immediate & $73.3(37.1)$ & $84.3(41.0)$ & 7.16 & 1,15 & 0.017 & 0.317 & 0.317 & 0.3 & $11 / 17$ \\
\hline & Delay & $23.3(12.2)$ & $25.4(14.3)$ & 2.22 & 1,13 & 0.160 & 0.103 & 0.103 & 0.2 & $9 / 15$ \\
\hline \multirow[t]{5}{*}{ Sensori-motor skills } & Imitation hand position & $10.5(4.7)$ & $11.5(4.6)$ & 2.55 & 1,13 & 0.134 & 0.636 & 0.636 & 0.2 & $10 / 15$ \\
\hline & $\begin{array}{l}\text { Speed in Visuomotor } \\
\text { precision }\end{array}$ & $108.4(48.6)$ & $116.0(54.1)$ & 1.82 & 1,12 & 0.202 & 0.795 & 0.795 & 0.2 & $4 / 14$ \\
\hline & $\begin{array}{l}\text { Accuracy in Visuomotor } \\
\text { precision }\end{array}$ & $59.4(52.3)$ & $56.8(50.7)$ & 0.012 & 1,12 & 0.915 & 0.215 & 0.215 & 0.1 & $8 / 14$ \\
\hline & Block Construction & $8.0(2.5)$ & $9.8(2.3)$ & 28.29 & 1,13 & $0.001^{*}$ & 0.863 & 0.112 & 0.7 & $13 / 15$ \\
\hline & Arrows & $14.5(9.8)$ & $15.5(7.2)$ & 1.50 & 1,12 & 0.245 & 0.303 & 0.886 & 0.1 & $9 / 14$ \\
\hline
\end{tabular}

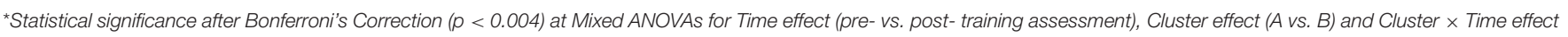

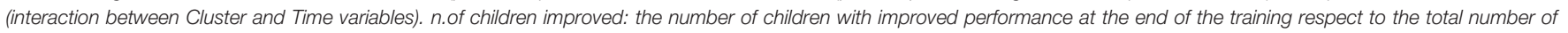
children who had completed the subtest.

functions is particularly important in children with pSD where the neuropsychological impairment may have a multi-level organization (36). Indeed, a training on WM may have direct effects on the EF impairment found in more than $50 \%$ of the children with pSD (36), and, at the same time, it may indirectly reduce visuo-spatial deficits, extensively documented in this clinical condition $(26,34)$.

In order to further understand how working memory training affects performance in children with $\mathrm{pSD}$, the study analyzed whether the training effects were correlated to the clinical characteristics of the sample. No correlations were found between chronological and gestational ages, intelligence level or gross motor functioning and on the trained WM outcomes (CogMed improvement index and Following the Instructions tests). These findings are in agreement with previous evidence describing gains in WM capacity after CogMed training in different clinical populations $(56,57)$, and across different ages $(48,55,63,78,79$, 85 ), and support that, within a specific CP form, improvements may be found regardless of clinical variability such as different levels of motor deficit, grades of prematurity and intelligence quotients, the latter, within the normal range.

Nevertheless, improvements in the untrained tasks were found to be variably related to clinical characteristics. The grossmotor functions severity was related to the improvement in visuo-motor tasks, visuo-spatial memory and visuo-perceptual abilities: as severity of gross motor impairment increased, children showed greater gains in visuo-motor and visual memory skills but smaller improvements in a visuo-perceptual task. On the basis of these findings, although exploratory and based on a small group, one could speculate that the different generalization effects of the WM training on the neuropsychological processes found impaired in children with $\mathrm{pSD}$, is related to the degree of gross motor disabilities.

Some methodological limitations of the present study should be pointed out. The data are based on a small sample of children with pSD due to periventricular leukomalacia and with average verbal intelligence, thus its findings must be confirmed in larger school-age samples, all trained with the same CogMed version. Moreover, since we used a comprehensive neuropsychological battery (NEPSY-II) to test EFs, a more finegrained analysis of specific processes and components accounting for performance may have been highlighted, if specific EF tasks had been implemented.

In spite of these limitations, the study underlines the importance for integrating cognitive trainings focused on EF in the rehabilitation schedules of children with $\mathrm{pSD}$ which are most frequently focused only on motor/psychomotor interventions.

In conclusion, this study suggests that a home-based working memory training in children with pSD has a beneficial effect on trained working memory tasks as well as a generalization effect on other visuo-spatial and executive function tasks, especially for those subcomponents requiring cognitive control and updating. This study extends Grunewaldt et al.'s results in premature children showing beneficial training effects also in premature children with cerebral palsy.

Although Randomized Control Trial longitudinal studies on larger sample, with statistically more rigorous and robust comparisons of primary and secondary outcomes are needed to confirm these findings, it is suggested that a home-based WM training is an effective intervention for children with pSD which as it contributes to reducing hospital stays, already prolonged for 
motor assessments and interventions, and preventing cognitive weaknesses negatively impacting educational achievement and social functions.

\section{DATA AVAILABILITY STATEMENT}

The raw data supporting the conclusions of this article will be made available by the authors, without undue reservation.

\section{ETHICS STATEMENT}

The studies involving human participants were reviewed and approved by Ethical Committee of the Fondazione Stella Maris Institute. Written informed consent to participate in this study was provided by the participants' legal guardian/next of kin.

\section{AUTHOR CONTRIBUTIONS}

MD: substantial contribution to the conception and design, acquisition, analysis and interpretation of data, and drafting. CP: substantial contribution to the conception and design, critical revision for important intellectual content, analysis and interpretation of data, and drafting. PB: substantial contribution to the conception and design, critical revision

\section{REFERENCES}

1. Baddeley AD. Working Memory. Oxford: Oxford University Press (1986).

2. Gathercole SE, Alloway TP, Willis C, Adams AM. Working memory in children with reading disabilities. J Exp Child Psychol. (2006) 93:265-81. doi: 10.1016/j.jecp.2005.08.003

3. Gathercole SE, Durling M, Evans S, Jeffcock E, Stone S. Working memory abilities and children's performance in laboratory analogues of classroom activities. Appl Cogn Psychol. (2008) 22:1019-37. doi: 10.1002/acp.1407

4. Oberauer K, Suß K-M, Wilhelm O, Wittmann WW. Which working memory functions predict intelligence? Intelligence. (2008) 36:641-52. doi: 10.1016/j.intell.2008.01.007

5. Jenks K, Moor J, Lieshout E. Arithmetic difficulties in children with cerebral palsy are related to executive function and working memory. J Child Psychol Psychiatry. (2009) 50:824-33. doi: 10.1111/j.1469-7610.2008.02031.x

6. Diamond A, Lee K. Interventions shown to aid executive function development in children 4 to 12 years old [Special section]. Science. (2011) 333:959-63. doi: 10.1126/science. 1204529

7. Diamond A. Executive functions. Annu Rev Psychol. (2012) 64:135-68. doi: 10.1146/annurev-psych-113011-143750

8. Wass SV. Applying cognitive training to target executive functions during early development. Child Neuropsychol. (2015) 21:150-66. doi: 10.1080/09297049.2014.882888

9. Miyake A, Friedman NP, Emerson MJ, Witzki AH, Howerter A. The unity and diversity of executive functions and their contributions to complex "Frontal Lobe" tasks: a latent variable analysis. Cogn Psychol. (2000) 41:49100. doi: 10.1006/cogp.1999.0734

10. Lehto JE, Juujärvi P, Kooistra L, Pulkkinen L. Dimensions of executive functioning: evidence from children. Br J Dev Psychol. (2003) 21:59-80. doi: 10.1348/026151003321164627

11. Friedman NP, Miyake A. Unity and diversity of executive functions: individual differences as a window on cognitive structure. Cortex. (2017) 86:186-204. doi: 10.1016/j.cortex.2016.04.023 for important intellectual content analysis and interpretation of data, and drafting. GS: critical revision for important intellectual content analysis, accountable for ensuring questions related to accuracy or integrity of any part of the work are appropriately investigated and resolved, and final approval. MD'O, SP, and ES: acquisition, analysis and interpretation of data. AC: substantial contribution to the conception and design and critical revision for important intellectual content. AG: critical revision for important intellectual content. GC: critical revision for important intellectual content, accountable for ensuring questions related to accuracy or integrity of any part of the work are appropriately investigated and resolved, and final approval. All authors contributed to the article and approved the submitted version.

\section{FUNDING}

This research was supported by RC 2014 and 2019 of the Italian Ministry of Health and Grant 5x1000 of same years.

\section{ACKNOWLEDGMENTS}

The authors have stated that they had no interests which might be perceived as posing a conflict or bias. The authors would like to thank the children and their families for participating in the study. We also thank Miningful Studio for statistical advice.

12. Wass SV, Sherif G, Johnson MH. Training attentional control and working memory - is younger, better? Dev Rev. (2012) 32:360-87. doi: 10.1016/j.dr.2012.07.001

13. Spencer-Smith $\mathrm{M}$, Klingberg T. Benefits of a working memory training program for inattention in daily life: a systematic review and meta-analysis. PLoS ONE. (2016) 10:e0119522. doi: 10.1371/journal.pone.0167373

14. De La Fuente A, Xia S, Branch C, Li X. A review of attentiondeficit/hyperactivity disorder from the perspective of brain networks. Front Hum Neurosci. (2013) 7:192. doi: 10.3389/fnhum.2013.00192

15. Beauchamp MH, Anderson V. Cognitive and psychopathological sequelae of pediatric traumatic brain injury. Handb Clin Neurol. (2013) 112:913-20. doi: 10.1016/B978-0-444-52910-7.00013-1

16. Kudo MF, Lussier CM, Swanson HL. Reading disabilities in children: a selective meta-analysis of the cognitive literature. Res Dev Disabil. (2015) 40:51-62. doi: 10.1016/j.ridd.2015.01.002

17. Kapa LL, Plante E. Executive function in SLI: recent advances and future directions. Curr Dev Disord Rep. (2015) 2:245-52. doi: $10.1007 / \mathrm{s} 40474-015-0050-\mathrm{x}$

18. Bexkens A, Ruzzano L, Collot D’Escury-Koenigs AM, Van der Molen MW, Huizenga HM. Inhibition deficits in individuals with intellectual disability: a meta-regression analysis. J Intellect Disabil Res. (2013) 58:3-16. doi: $10.1111 /$ jir. 12068

19. Costanzo F, Varuzza C, Menghini D, Addona F, Gianesini T, Vicari S. Executive functions in intellectual disabilities: a comparison between Williams syndrome and Down syndrome. Res Dev Disabil. (2013) 34:1770-80. doi: 10.1016/j.ridd.2013.01.024

20. Chen SF, Chien YL, Wu CT, Shang CY, Wu YY, Gau SS. Deficits in executive functions among youths with autism spectrum disorders: an age-stratified analysis. Psychol Med. (2016) 46:1625-38. doi: 10.1017/S00332917150 02238

21. Bottcher L, Flachs EM, Uldall P. Attention and executive impairment in children with spastic cerebral palsy. Dev Med Child Neurol. (2009) 52:42-7. doi: 10.1111/j.1469-8749.2009.03533.x 
22. Bodimeade HL, Whittingham K, Lloyd O, Boyd RN. Executive function in children and adolescents with unilateral cerebral palsy. Dev Med Child Neurol. (2013) 55:926-33. doi: 10.1111/dmcn.12195

23. Rosenbaum P, Paneth N, Leviton A, Goldstein M, Bax M, Damiano D, et al. A report: the definition and classification of cerebral palsy April 2006. Dev Med Child Neurol Suppl. (2007) 49:8-14. doi: 10.1111/j.1469-8749.2007.tb 12610.x

24. Cioni G, Bertuccelli B, Boldrini A, Canapicci R, Fazzi B, Guzzetta A, et al. Correlation between visual function, neurodevelopmental outcome, and magnetic resonance imaging findings in infants with periventricular leukomalacia. Arch Dis Child Fetal Neonatal Ed. (2000) 82:134-40. doi: $10.1136 /$ fn.82.2.F134

25. Jacobson LK, Dutton GN. Periventricular leukomalacia: an important cause of visual and ocular motility dysfunction in children. Surv Ophthalmol. (2000) 45:1-13. doi: 10.1016/S0039-6257(00)00134-X

26. Fazzi E, Bova S, Giovenzana A, Signorini S, Uggetti C, Bianchi P. Cognitive visual dysfunctions in preterm children with periventricular leukomalacia. Dev Med Child Neurol. (2009) 51:974-81. doi: $10.1111 / j .1469-8749.2009 .03272 . x$

27. Guzzetta A, Tinelli F, Bancale A, Cioni G. Visual and oculomotordisorders. In: Ferrari A, Cioni G, editors. The Spastic Forms of Cerebral Palsy, A Guide to the Assessment of Adaptive Functions. Milan: Springer (2010). p. 115-39.

28. Pavlova MA, Krägeloh-Mann I. Limitations on the developing preterm brain: impact of periventricular white matter lesions on brain connectivity and cognition. Brain. (2013) 136:998-1011. doi: 10.1093/brain/aws334

29. Ito J, Araki A, Tanaka H, Tasaki T, Cho K, Miyamoto A. Assessment of visuoperceptual disturbance in children with spastic diplegia using measurements of the lateral ventricles on cerebral MRI. Dev Med Child Neurol. (1996) 38:496-502. doi: 10.1111/j.1469-8749.1996.tb12110.x

30. Sigurdardottir S, Eiriksdottir A, Gunnarsdottir E, Meintema M, Arnadottir U, Vik T. Cognitive profile in young Icelandic children with cerebral palsy. Dev Med Child Neurol. (2008) 50:357-62. doi: 10.1111/j.1469-8749.2008.02046.x

31. Schatz J, Craft S, White D, Park TS, Figiel GS. Inhibition of return in children with perinatal brain injury. J Int Neuropsychol Soc. (2001) 7:275-84. doi: 10.1017/S1355617701733012

32. White DA, Christ SE. Executive control of learning and memory in children with bilateral spastic cerebral palsy. J Int Neuropsychol Soc. (2005) 11:920-4. doi: $10.1017 /$ S1355617705051064

33. Korkman M, Mikkola K, Ritari N, Tommiska V, Salokorpi T, Haataja L, et al. Neurocognitive test profiles of extremely low birth weight five-year-old children differ according to neuromotor status. Dev Neuropsychol. (2008) 33:637-55. doi: 10.1080/87565640802254471

34. Pueyo R, Junque C, Vendrell P, Narberhaus A, Segarra D. Neuropsychologic impairment in bilateral cerebral palsy. Pediatr Neurol. (2009) 40:19-26. doi: 10.1016/j.pediatrneurol.2008.08.003

35. Pirila S, van derMeere J, Rantanen K, Jokiluoma M, Eriksson K. Executive functions in youth with spastic cerebral palsy. J Child Neurol. (2011) 26:81721. doi: $10.1177 / 0883073810392584$

36. Di Lieto MC, Brovedani P, Pecini C, Chilosi A, Belmonti V, Fabbro F. Spastic diplegia in preterm born children: executive function impairment and neuroanatomical correlates. Res Dev Disabil. (2017) 61:116-26. doi: 10.1016/j.ridd.2016.12.006

37. Scheck SM, Pannek K, Raffelt DA, Fiori S, Boyd RN, Rose SE. Structural connectivity of the anterior cingulate in children with unilateral cerebral palsy due to white matter lesions. Neuroimage Clin. (2015) 9:498-505. doi: 10.1016/j.nicl.2015.09.014

38. Novak I, Morgan C, Fahey M, Finch-Edmondson M, Galea C, Hines A, et al. State of the evidence traffic lights 2019: systematic review of interventions for preventing and treating children with cerebral palsy. Curr Neurol Neurosci Rep. (2020) 20:1-21. doi: 10.1007/s11910-020$1022-\mathrm{z}$

39. Blauw-Hospers CH, de Graaf-Peters VB, Dirks T, Bos AF, Hadders-Algra M. Does early intervention in infants at high risk for a developmental motor disorder improve motor and cognitive development? Neurosci Biobehav Rev. (2007) 31:1201-12. doi: 10.1016/j.neubiorev.2007.04.010

40. Ziviani J, Feeney R, Rodger S, Watter P. Systematic review of early intervention programmemes for children from birth to nine years who have a physical disability. Aust Occup Ther J. (2010) 57:210-23. doi: $10.1111 / j .1440-1630.2010 .00850 . x$

41. Butler C, Darrah J. Effects of neurodevelopmental treatment (NDT) for cerebral palsy: an AACPDM evidence report. Dev Med Child Neurol. (2001) 43:778-90. doi: 10.1017/S0012162201001414

42. Pennington L, Goldbart J, Marshall J. Speech and language therapy to improve the communication skills of children with cerebral palsy. Cochrane Database Syst Rev. (2004) 2:CD003466. doi: 10.1002/14651858.CD003466.pub2

43. Tuersley-Dixon L, Frederickson N. Conductive education: appraising the evidence. Educ Psychol Pract. (2010) 26:353-73. doi: 10.1080/02667363.2010.521309

44. Mak C, Whittingham K, Cunnington R, Boyd RN. MiYoga: a randomised controlled trial of a mindfulness movement programme based on hatha yoga principles for children with cerebral palsy: a study protocol. BMJ Open. (2017) 7:e015191. doi: 10.1136/bmjopen-2016-015191

45. Piovesana M, Ross S, Lloyd O, Whittingham K, Ziviani J, Ware RS, et al. Randomized controlled trial of a web-based multi-modal therapy program for executive functioning in children and adolescents with unilateral cerebral palsy. Disabil Rehabil. (2017) 39:2021-8. doi: 10.1080/09638288.2016. 1213899

46. Olsen PJ, Westerberg H, Klingberg T. Increased prefrontal and patietal activity after training of working memory. Nat Neurosci. (2004) 7:75-9. doi: $10.1038 / \mathrm{nn} 1165$

47. McNab F, Varrone A, Farde L, Jucaite A, Bystritsky P, Forssberg H, et al. Changes in cortical dopamine D1 receptor binding associated with cognitive training. Science. (2009) 323:800-2. doi: 10.1126/science.1166102

48. Brehmer Y, Rieckmann A, Bellander M, Westerberg H, Fischer H, Bäckman L. Neural correlates of training-related working-memory gains in old age. Neuroimage. (2011) 58:1110-20. doi: 10.1016/j.neuroimage.2011.06.079

49. Klingberg T, Fernell E, Olesen PJ, Johnson M, Gustafsson P, Dahlström K, et al. Computerized training of working memory in children with ADHD a randomized, controlled trial. J Am Acad Child Adolesc Psychiatry. (2005) 44:177-86. doi: 10.1097/00004583-200502000-00010

50. Beck SJ, Hanson CA, Puffenberger SS, Benninger KL, Benninger WB. A controlled trial of working memory training for children and adolescents with ADHD. J Clin Child Adolesc Psychol. (2010) 39:825-36. doi: 10.1080/15374416.2010.517162

51. Gibson BS, Gondoli DM, Johnson AC, Steeger CM, Dobrzenski BA, Morrissey RA. Component analysis of verbal versus spatial working memory training in adolescents with ADHD: a randomized, controlled trial. Child Neuropsychol. (2011) 17:546-63. doi: 10.1080/09297049.2010.551186

52. Chacko A, Bedard AC, Marks DJ, Feirsen N, Uderman JZ, Chimiklis A, et al. A randomized clinical trial of CogMed Working Memory Training in school-age children with ADHD: a replication in a diverse sample using a control condition. J Child Psychol Psychiatry. (2014) 55:247-55. doi: $10.1111 /$ jcpp. 12146

53. Lundqvist A, Grundström K, Samuelsson K, Rönnberg J. Computerized training of working memory in a group of patients suffering from acquired brain injury. Brain Inj. (2010) 24:1173-83. doi: 10.3109/02699052.2010.498007

54. Kidokoro H, Anderson PJ, Doyle LW, Woodward LJ, Neil JJ, Inder TE. Brain injury and altered brain growth in preterm infants: predictors and prognosis. Pediatrics. (2014) 134:444-53. doi: 10.1542/peds.2013-2336

55. Løhaugen GCC, Antonsen I, Håberg A, Gramstad A, Vik T, Brubakk $\mathrm{AM}$, et al. Computerized working memory training improves function in adolescents born at extremely low birth weight. J Pediatr. (2011) 158:555-61. doi: 10.1016/j.jpeds.2010.09.060

56. Grunewaldt KH, Lohaugen GC, Austeng D, Brubakk AM, Skranes J. Working memory training improves cognitive function in VLBW preschoolers. Pediatrics. (2013) 131:747-54. doi: 10.1542/peds.2012-1965

57. Grunewaldt KH, Skranes J, Brubakk AM, Lähaugen GC. Computerized working memory training has positive long-term effect in very low birthweight preschool children. Dev Med Child Neurol. (2016) 58:195-201. doi: $10.1111 / \mathrm{dmcn} .12841$

58. Lee CS, Pei J, Andrew GA, Kerns K, Rasmussen C. Effects of working memory training on children born preterm. App Neuropsychol Child. (2017) 6:281-96. doi: $10.1080 / 21622965.2016 .1161513$ 
59. Anderson PJ, Lee KJ, Roberts G, Spencer-Smith MM, Thompson DK, Seal ML, et al. Long-term academic functioning following cogmed working memory training for children born extremely preterm: a randomized controlled trial. $J$ Pediatr. (2018) 202:92-7. doi: 10.1016/j.jpeds.2018.07.003

60. Westerberg H, Jacobaeus H, Hirvikoski T, Clevberger P, Ostensson ML, Bartfai A, et al. Computerized working memory training after stroke-a pilot study. Brain Inj. (2007) 21:21-9. doi: 10.1080/02699050601148726

61. Jaeggi S, Buschkuehl M, Jonides J, Perrig W. Improving fluid intelligence with training on working memory. Proc Natl Acad Sci USA. (2008) 105:6829-33. doi: 10.1073/pnas.0801268105

62. Holmes J, Gathercole SE, Dunning DL. Adaptive training leads to sustained enhancement of poor working memory in children. Dev Sci. (2009) 12:9-15. doi: 10.1111/j.1467-7687.2009.00848.x

63. Thorell L, Lindqvist S, Bergman-Nutley S, Bohlin G, Klingberg T. Training and transfer effects of executive functions in preschool children. Dev Sci. (2009) 12:106-13. doi: 10.1111/j.1467-7687.2008.00745.x

64. Loosli SV, Buschkuehl M, Perrig WJ, Jaeggi SM. Working memory training improves reading processes in typically developing children. Child Neuropsychol. (2012) 18:62-78. doi: 10.1080/09297049.2011. 575772

65. Dunning DL, Holmes J, Gathercole SE. Does working memory training lead to generalized improvements in children with low working memory? A randomized controlled trial. Dev Sci. (2013) 16:915-25. doi: $10.1111 /$ desc. 12068

66. Fuentes A, Kerr EN. Maintenance effects of working memory intervention (Cogmed) in children with symptomatic epilepsy. Epilepsy Behav. (2017) 67:51-9. doi: 10.1016/j.yebeh.2016.12.016

67. Kadam P, Bhalerao S. Sample size calculation. Int J Ayurveda Res. (2010) 1:55-7. doi: 10.4103/0974-7788.59946

68. Faul F, Erdfelder E, Lang AG, Buchner A. G* Power 3:A flexible statistical power analysis program for the social, behavioral, and biomedical sciences. Behav Res Methods. (2007) 39:175-91. doi: 10.3758/BF03193146

69. Cohen, J. (1988). Statistical Power Analysis for the Behavioral Sciences. 2nd ed. Hillsdale: Lawrence Erlbaum.

70. Bax M, Goldstein M, Rosenbaum P, Leviton A, Paneth N, Dan B, et al. Executive committee for the definition of cerebral palsy: proposed definition and classification of cerebral palsy, April 2005. Dev Med Child Neurol. (2005) 47:571-6. doi: 10.1017/S001216220500112X

71. Eliasson AC, Beckung E, Rosenbaum P. The Manual Ability Classification System (MACS) for children with cerebral palsy: scale development and evidence of validity and reliability. Dev Med Child Neurol. (2006) 48:549-54. doi: 10.1017/S0012162206001162

72. Wechsler D. WPPSI-III, Wechsler Preschool and Primary Scale of Intelligence. 3rd ed. San Antonio, TX: Psychological Corporation (2002). doi: 10.1037/t15177-000

73. Wechsler D. WISC-III, Wechsler Intelligent Scale for Children. 3rd ed. New York, NY: Psychological Corporation (1992).
74. Wechsler D. WISC-IV, Wechsler Intelligence Scale for Children. 4th ed. San Antonio, TX: Psychological Corporation (2003). doi: 10.1037/t15174-000

75. Palisano R, Rosenbaum P, Walter S, Russell D, Wood E, Galuppi B. Development and reliability of a system to classify gross motor function in children with cerebral palsy. Dev Med Child Neurol. (1997) 39:214-23. doi: 10.1111/j.1469-8749.1997.tb07414.x

76. Alloway TP. Automated Working Memory Assessment Manual. London: Pearson (2007).

77. Brown CA, Lilford LJ. The stepped wedge trial design: a systematic review. BMC Med Res Methodol. (2006) 6:54. doi: 10.1186/1471-2288-6-54

78. Green CT, Long DL, Green D, Iosif AM, Dixon JF, Miller MR, et al. Will working memory training generalize to improve off-task behaviour in children with attention-deficit/hyperactivity disorder? Neurotherapeutics. (2012) 9:639-48. doi: 10.1007/s13311-012-0124-y

79. Klingberg T, Forssberg H, Westerberg H. Training of working memory in children with ADHD. J Clin Exp Neuropsychol. (2002) 24:781-9. doi: 10.1076/jcen.24.6.781.8395

80. Korkman M, Kirk U, Kemp SL. NEPSY II. Clinical and Interpretative Manual. San Antonio, TX: Psychological Corporation (2007).

81. Melby-Lervag M, Hulme C. Is working memory training effective? a meta-analytic review. Dev Psychol. (2013) 49:270-91. doi: 10.1037/ a0028228

82. Diamond A, Ling DS. Conclusions about interventions, programs, and approaches for improving executive functions that appear justified and those that, despite much hype, do not. Dev Cogn Neurosci. (2016) 18:34-48. doi: 10.1016/j.dcn.2015.11.005

83. Alloway TP, Alloway RG. Investigating the predictive roles of workingmemory and IQ in academic attainment. J Exp Child Psychol. (2010) 106:20-9. doi: 10.1016/j.jecp.2009.11.003

84. Moffitt TE, Arseneault L, Belsky D, Dickson N, Hancox RJ, Harrington H, et al. A gradient of childhood self-control predicts health, wealth, and public safety. Proc Natl Acad Sci USA. (2011) 108:2693-8. doi: 10.1073/pnas.1010076108

85. Gropper RJ, Gotlieb H, Kronitz R, Tannock R. Working memory training in college students with ADHD or LD. J Atten Disord. (2014) 18:331-45. doi: $10.1177 / 1087054713516490$

Conflict of Interest: The authors declare that the research was conducted in the absence of any commercial or financial relationships that could be construed as a potential conflict of interest.

Copyright (C) 2021 Di Lieto, Pecini, Brovedani, Sgandurra, Dell'Omo, Chilosi, Guzzetta, Perazza, Sicola and Cioni. This is an open-access article distributed under the terms of the Creative Commons Attribution License (CC BY). The use, distribution or reproduction in other forums is permitted, provided the original author(s) and the copyright owner(s) are credited and that the original publication in this journal is cited, in accordance with accepted academic practice. No use, distribution or reproduction is permitted which does not comply with these terms. 